\title{
Does change in positive affect mediate and/or moderate the impact of symptom distress on psychological adjustment after cancer diagnosis? A prospective analysis
}

\author{
Wai-Kai Hou ${ }^{\mathrm{a} *}$, Chi-Ching Law ${ }^{\mathrm{b}}$ and Yiu-Tung Fu ${ }^{\mathrm{b}}$ \\ ${ }^{a}$ Department of Psychology, University of Hong Kong, Hong Kong SAR, China; \\ ${ }^{b}$ Department of Clinical Oncology, Queen Elizabeth Hospital, Hong Kong SAR, China
}

(Received 2 July 2008; final version received 15 October 2008)

\begin{abstract}
Physical symptom distress is one of the commonest correlates of psychological adjustment in cancer patients. Positive affect (PA) can be a dynamic resource for patients to cope with the cancer-related physical demands. The present study examined whether differential patterns of change in PA were associated with anxiety and depressed mood, and whether PA modified the association between change in symptom distress and psychological distress in 215 Chinese people newly diagnosed with colorectal cancer (CRC). Participants completed measures of physical symptoms, PA, and anxiety and depression at diagnosis and again at 3-month follow-up. Multivariate analyses of covariance revealed that at follow-up, people reporting higher anxiety and depressed mood demonstrated loss in PA, whereas those reporting lower depressed mood demonstrated a gain in PA. Structural equation modelling revealed that change in PA significantly mediated and moderated the associations between increased symptom distress and anxiety and depressed mood. We conclude that in line with Hobfoll's conservation of resources theory, continuous physical symptom distress depletes PA of newly diagnosed cancer patients, resulting in higher levels of anxiety and depressed mood. Effectiveness of symptom management intervention could be enhanced by preserving or enhancing PA in patients.
\end{abstract}

Keywords: resilience; resource change; positive affect; Chinese; colorectal cancer

\section{Introduction}

Cancer comprises multiple demanding situations and exposes patients to a broad range of threats, burdens and potential losses (Alonzo, 2000; Baum \& Posluszny, 2001). Apart from understanding adjustment as a pathological process, a growing body of evidence suggests the importance of studying positive emotional experience as an independent adaptive pathway in cancer experience (Aspinwall \& MacNamara, 2005; Folkman, 2008; Folkman $\&$ Moskowitz, 2000). In the present study, positive affect (PA) refers to affective states that are pleasant (e.g. joy, peace and excitement), as opposite to unpleasant and possibly distressing negative affect (NA) including tension, anger and depression (Watson, Clark, \& Tellegen, 1988).

\footnotetext{
*Corresponding author. Email: houwaikai@gmail.com
} 
Among long-term survivors, higher levels of daily PA were associated with subsequent uplift of natural killer cell activity (Vitaliano et al., 1998). A study of colorectal cancer (CRC) patients also found that higher preoperative PA but not lower NA predicted shorter length of hospital stay after curative resection (Sharma, Sharp, Walker, \& Monson, 2008). Voogt et al. (2005) found that PA, but not NA, of patients with advanced cancer was lower than the age-matched population norm and predictive of anxiety and depression. Ritterband and Spielberger (2001) further demonstrated in prostate and breast cancer patients that the inverse association between PA and depressed mood was independent of the levels of NA.

The trajectory of PA during diagnosis and treatment has also been analysed in conjunction with physical and psychological distress. Thornton, Perez, and Meyerowitz (2004) found that in the year following radical prostectomy, older men demonstrated stable PA in conjunction with decreasing NA and gradual restoration to presurgical level of physical functioning. Decline in cancer-specific physical symptoms has also been found to predict decline in depressed mood and maintenance of PA among older patients with breast, prostate and colon cancer in the year follow diagnosis (Stommel, Kurtz, Kurtz, Given, \& Given, 2004).

None of the previous studies, however, clarifies the mechanism through which PA influences physical and mental health outcomes of cancer patients. In particular, although cancer-related physical symptom distress has been shown to be one of the commonest demands associated with psychological distress (Given, Given, \& Stommel, 1993; Portenoy et al., 1994; van't Spijker, Trijsburg, \& Duivenvoorden, 1997), very little is known about the role of PA in the association between physical and psychological distress.

\section{$P A$ as a psychological resource}

Fredrickson's (2001) broaden-and-build theory suggests that PA has the power of undoing negative physical and psychological sequelae of stressful situations. Laboratory studies of college students have demonstrated that PA promoted faster recovery from cardiovascular reactions after induction of NA (Fredrickson \& Levenson, 1998; Fredrickson, Mancuso, Baranigan, \& Tugade, 2000). The dynamic model of affect (Zautra, Smith, Affleck, \& Tennen, 2001) similarly proposes that although PA and NA represent relatively independent affective states in ordinary circumstances, PA becomes bipolar to NA and maintains well-being by reducing NA or psychological distress in stressful situations. Studies of women with chronic illnesses including fibromyalgia, osteoporosis and rheumatoid arthritis have found that PA contributed to lower levels of NA among these women both directly and indirectly by modulating the negative impact of elevated pain intensity (Strand et al., 2006; Zautra, Johnson, \& Davis, 2005). PA can be conceptualised as a psychological resource modulating the impact of symptom distress on psychological well-being of cancer patients.

\section{Resource loss, resource gain}

It is also important to analyse temporally how PA functions in the face of ongoing challenges and demands of cancer diagnosis and treatment. Conservation of resources (COR) theory proposes that resource change is the central mechanism driving adaptation (Hobfoll, 1998). People offset ongoing situational demands by mobilising existing psychological and environmental resources during stress processes. To the extent that 
they can avoid depletion of resources or gain resources, less psychological morbidity would be manifested.

Hobfoll, Johnson, Ennis, and Jackson (2003) found that in the face of continuous economic stress, loss and gain in perceived self-mastery and social support were associated, respectively, with higher and lower anger and depression among inner city Native American women. Specifically, continuous economic stress contributed to higher anger and depressed mood both directly and indirectly by altering their self-mastery and social support. Among community and clinically depressed samples, Holahan, Moos, Holahan, and Cronkite $(1999,2000)$ have also found that negative life change predicted loss in personality (i.e. self-confidence and easygoingness) and social (family relational quality) resources over extended period of years, and the association between negative life change and depressed mood was completely mediated by change in these resources.

\section{The present study}

This study aims to examine the nature of relationships among PA, physical symptom distress, and psychological distress in Chinese people newly diagnosed with CRC. As the People's Republic of China (PRC) modernises, CRC has become the third commonest

100 cancer in urban Shanghai (Jin et al., 1999) and the fastest increasing cancer in Qidong city in the past decades (Chen et al., 2006). In Hong Kong, the most developed city in the PRC, CRC accounts for over $16 \%$ of all cancer cases, ranking second in incidence and mortality (Hong Kong Cancer Registry, 2005). CRC is also the most frequent cancer among Chinese Singaporeans (Seow et al., 2004). The increasing incidence suggests a need to understand

105 correlates of adjustment so as to optimise clinical and psychosocial services among Chinese CRC patients. Two studies have developed outcome measures for physical and psychological symptomatology and health-related quality of life (QoL) and demonstrated that psychological morbidity among Chinese CRC is strongly related to general physical distress including lack of energy and pain, and gastrointestinal tract symptoms such as

110 stomach pain, gas and belch (Lam et al., in press; Law et al., 2007). However, no studies have investigated the mechanism through which symptom distress impacts Chinese CRC patients' adjustment outcomes.

To examine the impact of PA as a dynamic psychological resource for adjustment to symptom experience, we measure PA as an affective state as opposed to a stable, trait-like

115 characteristic (Fredrickson, 2001; Zautra et al., 2001). Based on Hobfoll's COR theory, we use a prospective study with two time points, $\sim 3$ months apart to examine (1) whether change in symptom distress alters PA, (2) whether change in PA influences psychological distress in the form of anxiety and depression and (3) the extent to which change in PA mediates and/or moderates the impact of change in symptom distress on anxiety and 120 depression. The five hypotheses of this study are:

(1) Change in symptom distress is positively associated with anxiety and depressed mood at follow-up.

(2) Change in PA is negatively associated with anxiety and depressed mood at follow-up.

125 (3) Change in symptom distress is negatively associated with change in PA.

(4) The influence of change in symptom distress on anxiety and depressed mood is mediated by change in PA.

(5) The influence of change in symptom distress on anxiety and depressed mood is moderated by change in PA, such that the association between increase in 
symptom distress and higher levels of anxiety and depressed mood is stronger among patients reporting a loss in PA, whereas the association is weaker among those reporting a gain or maintenance in PA.

\section{Methods \\ Participants and procedure}

135 The present study is part of a larger longitudinal study on Chinese people with CRC in Hong Kong. Upon obtaining Ethics Committees' approvals from the University of Hong Kong and the Hospital Authority, recruitment was conducted in the largest Hong Kong government-funded oncology unit between July 2006 and March 2007. Inclusion criteria were (1) 21 years of age or older, (2) Cantonese fluency, (3) histological diagnosis of CRC

140 within 12 weeks' time and (4) no prior malignancies and associated therapies. Exclusion criteria included active axis I psychiatric disorders, linguistic/intellectual difficulties, brain metastasis, or serious medical condition(s).

Clinical oncologists introduced the purpose of the study to $263(86 \%)$ of the 305 suitable people in the oncology unit; voluntary participation and data confidentiality were

145 emphasised. Interviewer (W.K. Hou) then confirmed the eligibility of potential participants by referring to their hospital charts. After being fully apprised of the study, $234(89 \%)$ participants gave their written consent and completed the baseline assessments (Time 1) via face-to-face interview. Three months after the initial assessments, 215 participants were administered the follow-up assessments (Time 2) via structured 150 telephone interview. The present study included the participants who provided both Time 1 and Time 2 data.

The 215 participants ranged in age between 29 and 82 years $(M=64.28 ; \mathrm{SD}=10.58)$; $82(38 \%)$ were female. Most $(N=168,78 \%)$ were married, and lived with their spouse. Fifty-three $(25 \%)$ reported receiving no formal education, $70(33 \%)$ completed primary

155 education and $92(43 \%)$ completed at least secondary education. Of the 215 respondents, $113(53 \%)$ had colon cancer and $102(47 \%)$ had rectal cancer; $5(2 \%)$ had Stage I, 46 $(21 \%)$ Stage II, $124(58 \%)$ Stage III and 40 (19\%) Stage IV disease. Mean time since diagnosis was 36 days $(\mathrm{SD}=18.53)$. Most participants $(N=188,87 \%)$ have undergone curative or palliative resection, among which $61(28 \%)$ have a permanent colostomy. Over half of them $(N=131,61 \%)$ were receiving adjuvant therapies at Time 2.

\section{Measures}

\section{Demographic and medical characteristics}

A Chart Review Data Sheet was used to obtain diagnostic (site/sub-site, stage, time since diagnosis) and treatment (type and time of surgery and adjuvant therapies) information

165 from hospital charts. A standardised pro-forma was used to obtain demographic information including age, sex, marital status, current household size, education level, employment status and household income level.

\section{Physical symptom distress}

A checklist was developed by the authors to assess nine general and CRC-specific

170 symptoms: stomach-ache, gas/bloating, belch, pain in buttock, sleep problems, fatigue, pain, nausea and loss of appetite (Given, Given, \& Stommel, 1994; Law et al., 2007; 
Majumdar, Fletcher, \& Evans, 1999; Portenoy et al., 1994). Participants rated each symptom on a 4 -point scale $(0=$ not at all, $1=$ a little bit, $2=$ quite a bit, $3=$ very much $)$ to indicate presence and severity in the past week. A summation score was used (range $=0-27$ ). Alphas for the two administrations were 0.70 and 0.78 , respectively.

\section{Positive affect}

The 10-item PA subscale of Chinese Affect Scale (CAS-PA; Hamid \& Cheng, 1996) was used. The CAS-PA is linguistically and structurally equivalent to the Positive and Negative Affect Schedule (PANAS; Watson et al., 1988). Participants rated the frequency of 10 positive affective states in the previous 2 weeks on a 5 -point scale $(0=$ not at all, $1=$ rarely, $2=$ sometimes, $3=$ often, $4=$ all the time). A summation score was used (range $=0-40$ ). Reliability of the CAS-PA has been demonstrated in Chinese young and middle-age adults, with Cronbach's $\alpha$ 's above 0.85 (Hamid \& Cheng, 1996). Positive correlations with extraversion and optimism and negative correlations with physical and psychological morbidity demonstrated its validity (Hamid \& Cheng, 1996). Alphas for the CAS-PA were 0.90 and 0.92 , respectively, for the two administrations.

\section{Anxiety and depression}

Anxiety and depression were measured by using the 14-item Chinese hospital anxiety and depression scale (HADS; Leung, Ho, Kan, Hung, \& Chen, 1993). Seven items were 190 designed to measure anxiety and depressive symptoms, respectively. Participants answered each item on a 4-point scale (e.g. $0=$ not at all, $1=$ not very much, $2=$ quite a lot, $3=$ very much indeed). Anxiety (HADS-A) and depression (HADS-D) scores were calculated by summing across the seven items (range $=0-21$ ). The Chinese HADS was shown to be reliable (i.e. $\alpha>0.70)$ and valid in Chinese cancer populations (Ho, Chan, \& Ho, 2004).

195 Alphas for the HADS-A were 0.84 and 0.89 , respectively and for the HADS-D were 0.78 and 0.87 , respectively, on the two administrations.

\section{Creating comparison groups}

Following the method in Hobfoll et al. (2003) and Holahan et al. (1999, 2000), three comparison groups were created for PA: PA-loss, PA-stable and PA-gain. The CAS-PA scores at Time 1 were subtracted from the same scores at Time 2 to obtain a measure reflecting change in PA. The different scores were then used to create the three change groups, with the loss and gain groups being indicated by at least one half standard deviation of change on CAS-PA scores and the stable group being indicated by less than one half standard deviation of change on CAS-PA scores. This method of grouping can 205 distinguish conceptually unique upward and downward patterns of resource change (Hobfoll et al., 2003; Holahan et al., 1999, 2000). The same method was used to create three comparison groups for symptom distress: increased symptoms, stable symptoms and decreased symptoms.

\section{Statistical analyses}

210 Otherwise specified, all analyses were conducted by using SPSS (Version 14; SPSS Inc., Chicago, IL). To examine the impact of change in symptom distress (i.e. increased, decreased and stability) and the impact of change in PA (i.e. loss, gain and stability), 
three-way multivariate analyses of covariance (MANCOVAs) on Time 2 anxiety and depressed mood were performed, controlling for the initial levels of anxiety and

215 depressed mood and the demographic and medical variables (i.e. age, education level, employment status and income level) which were found to be significantly correlated with any of the study variables (Table 1). Bonferroni-adjusted $(p<0.025)$ planned comparisons then were used to examine (a) the relative impact of decreased versus increased symptom distress by contrasting the increased- and the stable-symptom groups and the decreased- and the stable-symptom groups and (b) the relative impact of loss versus gain in PA by contrasting the PA-loss and the stable groups and the PA-gain and the stable groups.

Structural equation modelling (SEM) with AMOS 7.0 (Arbuckle, 2006) was conducted to test the mediational and the moderator effect of change in PA in the association

225 between change in symptom distress and Time 2 psychological distress, controlling for the effects of the confounding variables. Separate models were conducted for anxiety and depressed mood because they have been found to be distinct adjustment problems in the year after cancer diagnosis (van't Spijker, Trijsburg, \& Duivenvoorden, 1997). Time 1 anxiety/depressed mood was included in the model as an exogenous variable in order to control for its effects on other variables; Time 2 anxiety/depressed mood was included as an endogenous variable. To obtain the measures of symptom change and PA change, the Time 2 scores were regressed on the Time 1 scores. The resulting residuals in the analyses were used as co-occurring endogenous variables in the SEM models. The Comparative Fit Index (CFI), Non-Normed Fit Index (NNFI), Incremental Fit Index (IFI) and

235 Standardised root mean square residual (SRMR) were used to evaluate the data-model fit because these fit indices provide a reliable evaluation of goodness-of-fit independent of sample size (Fan, Thompson, \& Wang, 1999). The model was accepted if all fit indices were over 0.95 and the SRMR under 0.08 (Hu \& Bentler, 1999). Shrout and Bolger's (2002) bootstrapping techniques were used to test the direct and indirect effects in

240 the mediational model. We produced 2000 bootstrap samples from the original data $(N=215)$ in order to derive bias-corrected $95 \%$ confidence intervals (BC 95\% CI), which have been shown to be accurate tests of mediational models in small sample size (MacKinnon, Lockwood, Hoffman, West, \& Sheets, 2002). The mediation effect was significant at $p<0.05$ if the BC $95 \%$ CI for the estimates did not include zero (Shrout \& 245 Bolger, 2002).

To examine the moderator effect of PA change in the association between symptom change and psychological distress, a cross-product interaction term for symptom change and PA change was included in the models as an exogenous variable. The interaction term was calculated by using the standard deviation scores of symptom change and PA change to

250 avoid multicollinearity of the interaction term with the two variables (Aiken \& West, 1991).

\section{Results}

\section{Descriptive statistics}

At Time 1, the 215 participants averaged $4.67(\mathrm{SD}=4.60)$ in anxiety and $6.81(\mathrm{SD}=4.75)$ in depressed mood. Using a cutoff HADS subscale score of eight to index cases/doubtful 255 cases of anxiety or depressed mood (Trask, 2004), 26\% of the participants $(N=55 ; 95 \%$ $\mathrm{CI}= \pm 5.83 \%)$ indicated clinically significant anxiety, $41 \%(N=87 ; 95 \% \mathrm{CI}= \pm 6.56 \%)$ indicated clinically significant depressed mood, and $20 \%(N=42 ; 95 \% \mathrm{CI}= \pm 5.30 \%)$ indicated comorbid anxiety and depressed mood. At Time 2, the participants averaged 


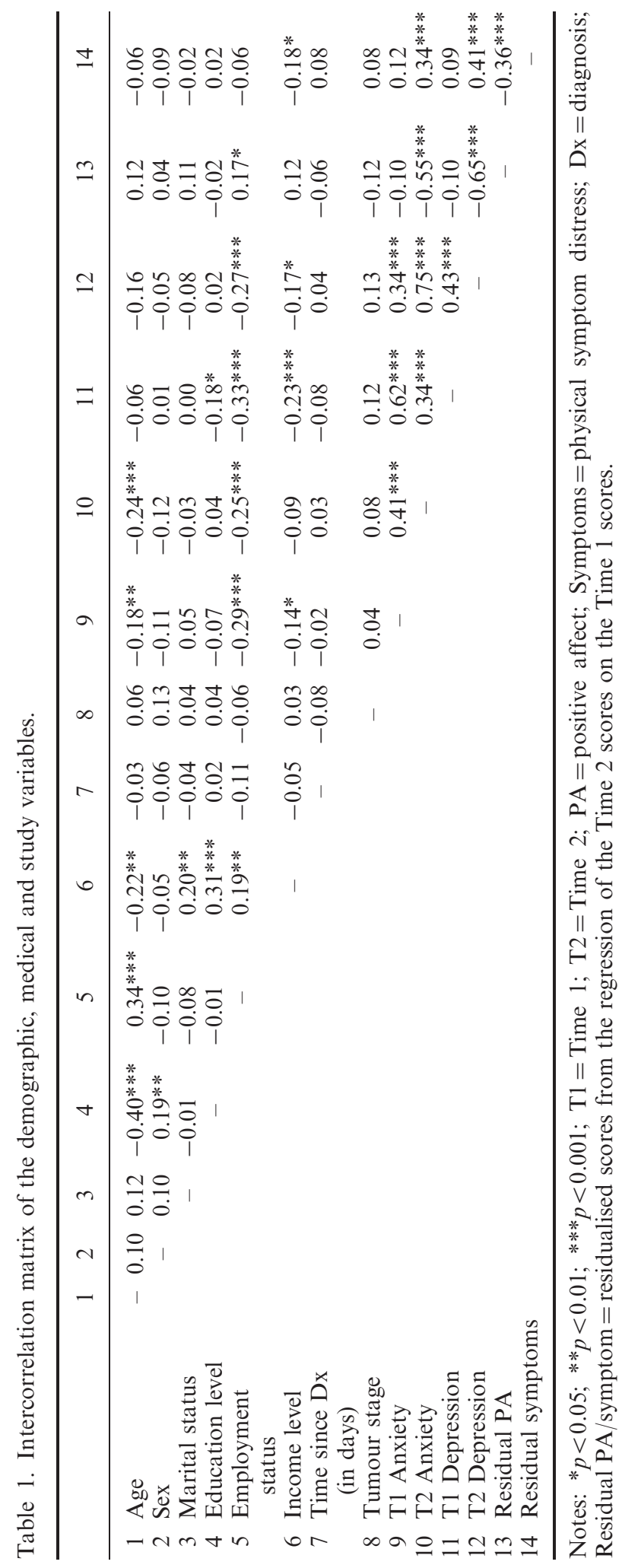


$4.35(\mathrm{SD}=4.91)$ in anxiety and $5.73(\mathrm{SD}=5.19)$ in depressed mood. Twenty-two percent of the participants $(N=48 ; 95 \% \mathrm{CI}= \pm 5.57 \%)$ indicated clinically significant anxiety, $31 \%(N=67 ; 95 \% \mathrm{CI}= \pm 6.19 \%)$ indicated clinically significant depressed mood, and $17 \%(N=36 ; 95 \% \mathrm{CI}= \pm 4.99 \%)$ indicated comorbid anxiety and depressed mood. Correlations among the Time 1 and Time 2 scores on the study variables are summarised in Table 2.

\section{Impact of change in symptom distress}

Means and standard deviations of anxiety and depressed mood for the change groups are summarised in Table 3. In the MANCOVA for change in symptom distress, the overall $F$ was significant, $F(4,410)=3.63, p<0.05$. It was also significant for anxiety, $F(2,206)=4.32$, $p<0.05$, and depressed mood, $F(2,206)=7.35, p<0.001$. Planned comparisons showed

Table 2. Intercorrelation matrix of the Time 1 and Time 2 scores on the study variables.

\begin{tabular}{|c|c|c|c|c|c|c|c|c|c|}
\hline & & 1 & 2 & 3 & 4 & 5 & 6 & 7 & 8 \\
\hline 1 & T1 Anxiety & - & $0.41 * * *$ & $0.62 * * *$ & $0.34 * * *$ & $-0.54 * * *$ & $-0.33 * * *$ & $-0.33 * * *$ & $-0.21 * *$ \\
\hline 2 & T2 Anxiety & & - & $0.34 * * *$ & $0.75 * * *$ & $-0.29 * * *$ & $-0.62 * * *$ & $0.24 * * *$ & $0.40 * * *$ \\
\hline 3 & T1 Depression & & & - & $0.43 * * *$ & $-0.72 * * *$ & $-0.44 * * *$ & $0.48 * * *$ & $0.22 * *$ \\
\hline 4 & T2 Depression & & & & - & $-0.43^{* * *}$ & $-0.78 * * *$ & $0.28 * * *$ & $0.48 * * *$ \\
\hline 5 & T1 PA & & & & & - & $-0.49 * * *$ & $-0.37 * * *$ & $-0.28 * * *$ \\
\hline 6 & T2 PA & & & & & & - & $-0.27 * * *$ & $-0.47 * * *$ \\
\hline 7 & T1 Symptoms & & & & & & & - & $0.31 * * *$ \\
\hline 8 & T2 Symptoms & & & & & & & & - \\
\hline
\end{tabular}

Notes: $\quad * * p<0.01 ; \quad * * * p<0.001 ; \quad \mathrm{T} 1=$ Time $\quad 1 ; \quad \mathrm{T} 2=$ Time $\quad 2 ; \quad \mathrm{PA}=$ positive $\quad$ affect; Symptoms $=$ physical symptom distress.

Table 3. Means and SDs of anxiety and depression for change groups.

\begin{tabular}{|c|c|c|c|c|c|c|c|c|}
\hline \multirow[b]{3}{*}{ Group } & \multicolumn{4}{|c|}{ Anxiety } & \multicolumn{4}{|c|}{ Depressed mood } \\
\hline & \multicolumn{2}{|c|}{ Time 1} & \multicolumn{2}{|c|}{ Time 2} & \multicolumn{2}{|c|}{ Time 1} & \multicolumn{2}{|c|}{ Time 2} \\
\hline & $M$ & $\mathrm{SD}$ & $M$ & $\mathrm{SD}$ & $M$ & $\mathrm{SD}$ & $M$ & $\mathrm{SD}$ \\
\hline \multicolumn{9}{|c|}{ Symptom distress change } \\
\hline Increased $(N=40)$ & 4.43 & 5.00 & 6.08 & 5.24 & 8.60 & 4.44 & 8.08 & 5.95 \\
\hline Stable $(N=110)$ & 4.50 & 4.39 & 3.85 & 4.82 & 5.99 & 4.64 & 4.93 & 4.59 \\
\hline Decreased $(N=65)$ & 5.12 & 4.73 & 4.15 & 4.68 & 6.15 & 4.82 & 5.66 & 5.29 \\
\hline \multicolumn{9}{|l|}{ Positive affect change } \\
\hline Loss $(N=52)$ & 4.25 & 3.79 & 6.96 & 5.42 & 5.54 & 4.01 & 8.25 & 4.75 \\
\hline Stable $(N=85)$ & 4.19 & 4.64 & 4.25 & 5.30 & 6.46 & 4.96 & 6.02 & 5.83 \\
\hline Gain $(N=78)$ & 5.49 & 4.97 & 2.73 & 3.09 & 8.04 & 4.73 & 3.74 & 3.79 \\
\hline
\end{tabular}

Notes: Increased/Gain $=$ at least $0.50 \mathrm{SD}$ of increase in scores between Time 1 and Time 2; Stable $=$ less than 0.50 SD of change in scores between Time 1 and Time 2; Decreased $/$ Loss $=$ at least $0.50 \mathrm{SD}$ of decrease in scores between Time 1 and Time 2.

$\mathrm{SD}=$ standard deviation. 
270 that the increased-symptoms group had higher levels of depressed mood ( $M$ difference $=3.15$, $p=0.001)$ and marginally higher levels of anxiety ( $M$ difference $=2.23, p=0.027)$ than the stable-symptoms group. Decreased- and stable-symptoms groups reported similar levels of anxiety $(M$ difference $=-1.92, p=0.11)$ and depressed $\operatorname{mood}(M$ difference $=0.73, p=0.98)$ (Table 4).

\section{Impact of PA-loss and PA-gain}

In the MANCOVA for change in PA, the overall $F$ was significant, $F(4,410)=15.36$, $p<0.001$. Analysis was also significant for anxiety, $F(2,206)=23.12, p<0.001$, and depressed mood, $F(2,206)=29.62, p<0.001$. Planned comparisons showed that the PA-loss group had higher levels of anxiety ( $M$ difference $=2.71, p=0.003)$ and 280 depressed mood $(M$ difference $=2.23, p=0.025)$ than the PA-stable group. The PA-gain group had similar levels of anxiety $(M$ difference $=-1.52, p=0.11)$ but significantly lower levels of depressed mood ( $M$ difference $=-2.28, p=0.008$ ) than the PA-stable group (Table 4).

\section{Structural equation modelling}

285 All fit indices and SRMR for the two models exceeded cutoff criteria, indicating good data-model fit. The models were just-identified, with zero degrees of freedom and a chisquare value of zero. Controlling for the effect of Time 1 anxiety and the confounding variables, change in PA $(\beta=-0.46, p<0.001)$ but not change in symptom distress $(\beta=0.13, p>0.05)$ significantly predicted Time 2 anxiety. The bootstrap BC 95\% CI did

290 not include zero for the model (0.11-0.23), suggesting a significant mediational effect of PA change in the association between symptom change and anxiety $(\beta=0.16, p<0.001)$. Taken together, the association between symptom change and Time 2 anxiety was completely mediated by PA change, such that increased symptom distress was associated with a decline in PA and, in turn, predicted higher levels of Time 2 anxiety. Controlling for 295 the effect of Time 1 depressed mood and the confounding variables, both change in symptom distress $(\beta=0.18, p<0.001)$ and change in PA $(\beta=-0.54, p<0.001)$

Table 4. Results of the Bonferroni-adjusted planned comparisons of anxiety and depressed mood.

\begin{tabular}{lccrr}
\hline Dependent variable & Comparison & Mean difference & $P$ & Effect size \\
\hline \multirow{3}{*}{ Anxiety } & Symptom distress change & & & \\
& Increased vs. Stable & 2.230 & 0.027 & 0.452 \\
Depressed mood & Decreased vs. Stable & 0.304 & 1.000 & 0.063 \\
& Increased vs. Stable & 3.147 & 0.001 & 0.632 \\
& Decreased vs. Stable & 0.734 & 0.975 & 0.150 \\
Anxiety & Positive affect change & & & \\
Depressed mood & Loss vs. Stable & 2.714 & 0.003 & 0.507 \\
& Gain vs. Stable & -1.516 & 0.106 & -0.347 \\
& Loss vs. Stable & 2.227 & 0.025 & 0.409 \\
& Gain vs. Stable & -2.280 & 0.008 & -0.460 \\
\hline
\end{tabular}

Note: Mean difference is significant at $p<0.025$. 
significantly predicted Time 2 depressed mood. The bootstrap BC 95\% CI did not include zero for the model (0.13-0.26), suggesting significant mediational effect of PA change in the association between symptom change and depressed mood $(\beta=0.19, p<0.001)$. The association between symptom change and Time 2 depressed mood was partially mediated by PA change, such that increased symptom distress was associated with higher levels of Time 2 depressed mood partially due to its negative impact on PA (i.e. a decline in PA). The standardised estimates of the two structural models are summarised in Figures 1 and 2.

The interaction term for symptom change and PA change significantly predicted Time 2 anxiety $(\beta=-0.19,95 \% \mathrm{CI}=-1.57$ to $-0.42, p<0.01)$ and Time 2 depressed $\operatorname{mood}(\beta=-0.16,95 \% \mathrm{CI}=-1.42$ to $-0.33, p<0.01)$, indicating that PA change significantly moderated the association between symptom change and Time 2 psychological distress. The negative interaction effects indicated that the association between increased symptom distress and higher Time 2 anxiety and depressed mood was stronger in the context of a loss in PA, while the association was weaker in the context of a gain or maintenance in PA.

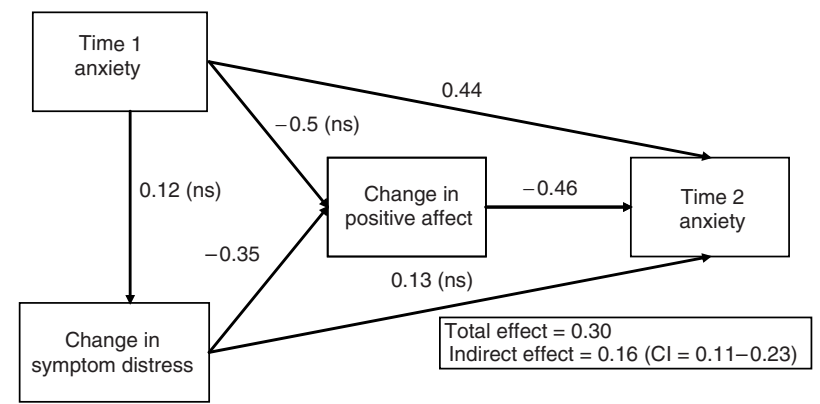

Figure 1. Standardised estimates of the structural equation model of relationships among change in physical symptom distress, change in positive affect and anxiety. ns $=$ non-significant $(p \geq 0.05)$; the covariates (i.e. age, education level, employment status and income level) are not illustrated in the figure.

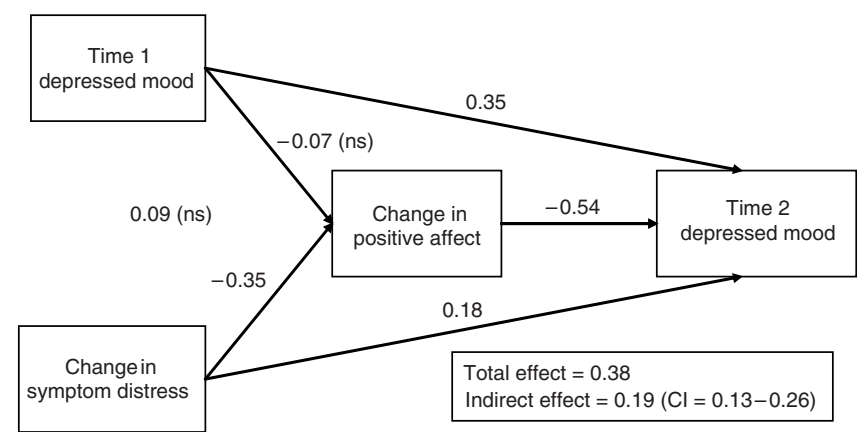

Figure 2. Standardised estimates of the structural equation model of relationships among change in physical symptom distress, change in positive affect and depressed mood. ns = non-significant $(p \geq 0.05)$; the covariates (i.e. age, education level, employment status and income level) are not illustrated in the figure. 


\section{Discussion}

The primary goal of the present study is to investigate whether, and how PA impacts adjustment to cancer diagnosis and treatment. In particular, we are interested in the mediational and the moderator role of change in PA in the association between change in physical symptom distress and psychological outcomes. Pertaining to our first hypothesis, compared to people reporting stable symptom distress, people reporting increased symptom distress had significantly higher anxiety and depressed mood at 3-month followup. Consistent with our second hypothesis, compared to people reporting stable PA, people losing PA reported higher anxiety and depressed mood while those gaining PA reported lower depressed mood at follow-up. Our third and fourth hypotheses were supported by the SEM results. The positive association between change in symptom distress and Time 2 anxiety was completely mediated by change in PA, whereas the positive association between change in symptom distress and Time 2 depression was partially mediated by change in PA. Pertaining to our fifth hypothesis, the positive associations between change in symptom distress and Time 2 anxiety and depressed mood were significantly moderated by change in PA.

Previous studies of cancer populations have reported concurrent relationships between

330 PA and psychological distress on the one hand (Ritterband \& Speilberger, 2001; Voogt et al., 2005) and change in PA during year after diagnosis on the other (Stommel et al., 2004; Thornton et al., 2004). However, none of these studies have examined the role of PA as a psychological resource in adjustment to cancer over time. Based on the broaden-andbuild theory (Fredrickson, 2001) and the dynamic model of affect (Zautra et al., 2001), we 335 conceptualised PA as a psychological resource and found that both loss and gain in PA during the immediate period of cancer diagnosis are significant predictors of anxiety and depressed mood.

The present study also goes beyond existing research by utilising Hobfoll's COR theory to explain psychosocial adjustment to cancer. Previous studies have examined change in 340 cancer patients' optimism (e.g. Pinquart, Fröhlich, \& Sibereisen, 2007; Schou, Ekber, Sandviki, Hjermstad, \& Ruland, 2005), self-esteem (e.g. Vinokur, Threatt, VinokurKaplan, \& Satariano, 1990), mastery (e.g. Henselmans, Sanderman, Baas, Smink, \& Ranchor, in press; Revenson, Wollman, \& Felton, 1983), and perceived social support (Bolger, Foster, Vinokur, \& Ng, 1996) in the year after diagnosis. However, these studies aggregated data across participants, precluding identification of differential patterns of change in these psychosocial resources. In addition, only few of them (Bolger et al., 1996; Pinquart et al., 2007) clarified the underlying mechanism of the impact of resource change.

Consistent with the corollary that the negative impact of demanding situations on mental health is due in part or entirely to depletion of resources (Hobfoll, 1998), we show 350 that during the immediate period of diagnosis, continuous symptom distress is associated with higher levels of anxiety entirely because of its depletive effect on PA of Chinese CRC patients, whereas continuous symptom distress contributes to higher depressed mood both directly and indirectly by eroding their PA. The positive association between increase in symptom distress and anxiety and depressed mood is stronger among patients showing a

355 loss in PA, whereas the association is weaker among those showing a gain or maintenance in PA.

These findings are consistent with a growing body of cross-sectional and prospective evidence on the centrality of resource change in adjustment to natural disaster (Norris \& Kaniasty, 1996; Smith \& Freedy, 2000), terrorist threat (Bonanno, Galea, Bucciarelli, \& 360 Vlahov, 2007; Hobfoll, Tracy, \& Galea, 2006), and economic stress (Hobfoll et al., 2003; 
Wells, Hobfoll, \& Lavin, 1999). The buffering effect for PA on the association between change in physical symptoms and psychological distress was also consistent with the current literature. Higher PA has been found to predict fewer physical symptoms among healthy people (Benyamini, Idler, Leventhal, \& Leventhal, 2000; Kunzmann, Little, \&

365 Smith, 2000) and buffer the negative impact of symptom distress on psychological wellbeing among chronic patients (Strand et al., 2006; Zautra et al., 2005). A follow-up study is currently underway to examine whether loss and gain in PA show sustainable impact a year after diagnosis.

Several limitations of the present study should be noted. First, the participants were a convenience sample from one tertiary clinic in Hong Kong, restricting generalisability of the findings to other Chinese cancer populations. Second, the measure of physical symptoms was not a formal instrument and was selected for parsimony. Moreover, we were unable to fully control for ongoing treatment and NA, which might influence experience of symptoms and PA, although we found that receipt of adjuvant therapies was only weakly correlated with both physical and psychological distress and there is consistent evidence that PA benefits cancer patients' psychological well-being independent of NA (Ritterband \& Spielberger, 2001; Sharma et al., 2008; Voogt et al., 2005).

Despite these limitations, the present study contributes to advancing psychooncological research in Chinese populations. Currently, very little is known about the psychological well-being of Chinese cancer patients. A study of Chinese women with gynaecologic cancers has demonstrated a link between satisfaction with life and social relationships; however, a small sample size $(N=62)$ and the use of non-validated measure (i.e. a single item) to assess well-being limit generalisability and conclusiveness of the findings (Molassiotis, Chan, Yam, \& Chan, 2000). We extend previous research by using 385 an adequate sample of Chinese patients $(N=215)$, one of the largest samples in psychooncology research of Chinese. The use of validated measures to assess PA also enhances conclusiveness of the present findings and facilitates replication and extension in future studies.

This study also informs psychosocial research and care for Chinese CRC populations and suggests a feasible direction for delivering appropriate service to newly diagnosed cancer patients. Recognising loss in PA as a significant mediator between symptom experience and psychological distress, enhancement of PA should be one of the components in symptom management. Recent studies have demonstrated that teaching strategies to identify and attain desirable goals significantly heightened PA in college

395 (Sheldon, Kasser, Smith, \& Share, 2002) and middle-age samples (MacLeod, Coates, \& Hetherton, 2007). Practicing regular grateful thinking has also been found to effectively increase PA of college students (Emmons \& McCullough, 2003). Efficacy of symptom management interventions for newly diagnosed cancer patients could be strengthened by incorporating education programs on goal attainment and regular grateful thinking.

400 Future studies should also identify further pathways that increase PA among cancer patients.

\section{References}

Aiken, L.S., \& West, S.G. (1991). Multiple regression: Testing and interpreting interactions. Newbury Park, CA: Sage.

405 Alonzo, A.A. (2000). The experience of chronic illness and post-traumatic stress disorder: The consequences of cumulative adversity. Social Science and Medicine, 50, 1475-1484. 
Arbuckle, J.L. (2006). Amos (Version 7.0) [Computer Program]. Chicago: SPSS.

Aspinwall, L.G., \& MacNamara, A. (2005). Taking positive changes seriously: Toward a positive psychology of cancer survivorship and resilience. Cancer, 104(Suppl. 11), 2549-2556.

Baum, A., \& Posluszny, D.M. (2001). Traumatic stress as a target for intervention with cancer patients. In A. Baum, \& B.L. Andersen (Eds.), Psychosocial interventions for cancer (pp. 143-174). Washington, DC: American Psychological Association.

Benyamini, Y., Idler, E.L., Leventhal, H., \& Leventhal, E.A. (2000). Positive affect and function as influences on self assessments of health: Expanding our view beyond illness and disability. Journal of Gerontology: Psychological Sciences, 55B, 107-116.

Bolger, N., Foster, M., Vinokur, A.D., \& Ng, R. (1996). Close relationships and adjustment to a life crisis: The case of breast cancer. Journal of Personality and Social Psychology, 70, 283-294.

Bonanno, G.A., Galea, S., Bucciarelli, A., \& Vlahov, D. (2007). What predicts psychological resilience? The role of demographics, resources, and life stress in the aftermath of disaster. Journal of Consulting and Clinical Psychology, 75, 671-682.

Chen, J.G., Zhu, J., Parkin, D.M., Zhang, Y.H., Lu, J.H., Zhu, Y.R., et al. (2006). Trends in the incidence of cancer in Qidong, China, 1978-2002. International Journal of Cancer, 119, $1447-1454$.

Emmons, R.A., \& McCullough, M.E. (2003). Counting blessings versus burdens: An experimental investigation of gratitude and subjective well-being in daily life. Journal of Personality and Social Psychology, 84, 377-389.

Fan, X., Thompson, B., \& Wang, L. (1999). The effects of sample size, estimation methods, and model specification on SEM fit indices. Structural Equation Modeling, 6, 56-83.

Folkman, S. (2008). The case for positive emotions in the stress process. Anxiety, Stress, and Coping, $21,3-14$.

Folkman, S., \& Moskowitz, J.T. (2000). Positive affect and the other side of coping. American Psychologist, 55, 647-654.

Fredrickson, B.L. (2001). The role of positive emotions in positive psychology: The broaden-andbuild theory of positive emotions. American Psychologist, 56, 218-226.

435 Fredrickson, B.L., \& Levenson, R.W. (1998). Positive emotions speed recovery from the cardiovascular sequelae of negative emotions. Cognition and Emotion, 12, 191-220.

Fredrickson, B.L., Mancuso, R.A., Baranigan, C., \& Tugade, M.M. (2000). The undoing effect of positive emotions. Motivation and Emotion, 24, 237-258.

Given, C.W., Given, B.A., \& Stommel, M. (1994). The impact of age, treatment and symptoms on the physical and mental health of cancer patients: A longitudinal study. Cancer, 74, $2128-2138$.

Hamid, P.N., \& Cheng, S.T. (1996). The development and validation of an index of emotional disposition and mood state: The Chinese Affect Scale. Education and Psychological Measurement, 56, 995-1014.

Henselmans, I., Sanderman, R., Baas, P.C., Smink, A., Ranchor, A.V. (in press). Personal control after a breast cancer diagnosis: Stability and adaptive value. Psycho-Oncology.

Ho, S.M.Y., Chan, C.L.W., \& Ho, R.T.H. (2004). Posttraumatic growth in Chinese cancer survivors. Psycho-Oncology, 13, 377-389.

Hobfoll, S.E. (1998). Stress, culture, and community: The psychology and philosophy of stress. New York: Plenum Press.

Hobfoll, S.E., Johnson, R.J., Ennis, N., \& Jackson, A.P. (2003). Resource loss, resource gain, and emotional outcomes among inner city women. Journal of Personality and Social Psychology, 84, 632-643.

Hobfoll, S.E., Tracy, M., \& Galea, S. (2006). The impact of resource loss and traumatic growth on probable PTSD and depression following terrorist attacks. Journal of Traumatic Stress, 19, $867-868$.

Holahan, C.J., Moos, R.H., Holahan, C.K., \& Cronkite, R.C. (1999). Resource loss, resource gain, and depressive symptoms: A 10-year model. Journal of Personality and Social Psychology, 77, $620-629$. 
Holahan, C.J., Moos, R.H., Holahan, C.K., \& Cronkite, R.C. (2000). Long-term posttreatment functioning among patients with unipolar depression: An integrative model. Journal of Consulting and Clinical Psychology, 68, 226-232.

Hong Kong Cancer Registry. (2005). Cancer Statistics 2002. Hong Kong: Hong Kong Hospital Authority.

465 Hu, L., \& Bentler, P.M. (1999). Cutoff criteria for fit indexes in covariance structure analysis: Conventional criteria versus new alternatives. Structural Equation Modeling, 6, 1-55.

Jin, F., Devesa, S.S., Chow, W.H., Zheng, W., Ji, B.T., Fraumeni, J.F., et al. (1999). Cancer incidence trends in urban Shanghai, 1972-1994: An update. International Journal of Cancer, 83, 435-440.

470 Kunzmann, U., Little, T.D., \& Smith, J. (2000). Is age-related stability of subjective wellbeing a paradox? Cross-sectional and longitudinal evidence from the Berlin Aging Study. Psychology and Aging, 15, 511-526.

Lam, W.W.T., Law, C.C., Fu, Y.T., Wong, K.H., Chang, V.T., \& Fielding, R. (in press). New insights in symptom assessment: The Chinese versions of the Memorial Symptom Assessment Scale Short Form (MSAS-SF) and the condensed MSAS (CMSAS). Journal of Pain and Symptom Management.

Law, C.C., Lam, W.W.T., Fu, Y.T., Wong, K.H., Sprangers, M.A.G., \& Fielding, R. (2008). Validation of the Chinese version of the EORTC colorectal cancer-specific quality-of-life questionnaire module (QLQ-CR38). Journal of Pain and Symptom Management, 35, 203-213.

Leung, C.M., Ho, S., Kan, C.S., Hung, C.H., \& Chen, C.N. (1993). Evaluation of the Chinese version of the hospital anxiety and depression scale. A cross-cultural perspective. International Journal of Psychosomatics, 40, 29-34.

MacKinnon, D.P., Lockwood, C.M., Hoffman, J.M., West, S.G., \& Sheets, V. (2002). A comparison of methods to test mediation and other intervening variable effects. Psychological Methods, 7 , 83-104.

MacLeod, A.K., Coates, E., \& Hetherton, J. (2007). Increasing well-being through teaching goalsetting and planning skills: Results of a brief intervention. Journal of Happiness Studies, 9, 185-196.

Majumdar, S.R., Fletcher, R.H., \& Evans, A.T. (1999). How does colorectal cancer present? Symptoms, duration, and clues to location. American Journal of Gastroenterology, 94, 3039-3045.

Molassiotis, A., Chan, C.W.H., Yam, B.M.C., \& Chan, S.J. (2000). Quality of life in Chinese women with gynaecological cancers. Supportive Care in Cancer, 8, 414-422.

495 of the social support deterioration deterrence model. Journal of Personality and Social Psychology, 71, 498-511.

Pinquart, M., Fröhlich, C., \& Sibereisen, R.K. (2007). Change in psychosocial resources of younger and older cancer patients during chemotherapy. Psycho-Oncology, 16, 626-633.

Portenoy, R.K., Thaler, H.T., Kornblith, A.B., Lepore, J.M., Friedlander-Klar, H., Coyle, N., et al. (1994). Symptom prevalence, characteristics and distress in a cancer population. Quality of Life Research, 3, 183-189.

Revenson, T.A., Wollman, C.A., \& Felton, B.J. (1983). Social support as stress buffer for adult cancer patients. Psychosomatic Medicine, 45, 321-331.

Ritterband, L.M., \& Spielberger, C.D. (2001). Depression in a cancer patient population. Journal of Clinical Psychology in Medical Settings, 8, 85-93.

Seow, A., Koh, W.P., Chia, K.S., Shi, L.M., Lee, H.P., \& Shanmugaratnam, K. (2004). Trends in cancer incidence in Singapore 1968-2002. Singapore Cancer Registry Report No 6. Singapore: Singapore Cancer Registry.

Schou, I., Ekeberg, O., Ruland, C.M., Sandik, L., \& Karesen, R. (2004). Pessimism as a predictor of emotional morbidity one year following breast cancer surgery. Psycho-Oncology, 13(5), 309-320.

Sharma, A., Sharp, D.M., Walker, L.G., \& Monson, J.R. (2008). Patient personality predicts postoperative stay after colorectal cancer resection. Colorectal Disease, 10, 151-156. 
Sheldon, K.M., Kasser, T., Smith, K., \& Share, T. (2002). Personal goals and psychological growth: Testing an intervention to enhance goal-attainment and personality integration. Journal of Personality, 70, 5-31.

Shrout, P.E., \& Bolger, N. (2002). Mediation in experimental and nonexperimental studies: New procedures and recommendations. Psychological Methods, 7, 422-445.

Smith, B.W., \& Freedy, J.R. (2000). Psychosocial resource loss as a mediator of the effects of flood exposure on psychological distress and physical symptoms. Journal of Traumatic Stress, 13, 349-357.

Stommel, M., Kurtz, M.E., Kurtz, J.C., Given, C.W., \& Given, B.A. (2004). A longitudinal analysis of the course of depressive symptomatology in geriatric patients with cancer of the breast, colon, lung, or prostate. Health Psychology, 23, 564-573.

Strand, E.B., Zautra, A.J., Thoresen, M., Odegard, S., Uhlig, T., \& Finset, A. (2006). Positive affect as a factor of resilience in the pain-negative affect relationship in patients with rheumatoid arthritis. Journal of Psychosomatic Research, 60, 477-484.

Thornton, A.A., Perez, M.A., \& Meyerowitz, B.E. (2004). Patient and partner quality of life and psychosocial adjustment following radical prostatectomy. Journal of Clinical Psychology in Medical Settings, 11, 15-30.

530 Trask, P.C. (2004). Assessment of depression in cancer patients. Journal of the National Cancer Institute Monographs, 32, 80-92.

van’t Spijker, A., Trijsburg, R.W., \& Duivenvoorden, H.J. (1997). Psychological sequelae of cancer diagnosis: A meta-analytic review of 58 studies after 1980. Psychosomatic Medicine, 59, 280-293.

Vinokur, A.D., Threatt, B.A., Vinokur-Kaplan, D., \& Satariano, W.A. (1990). The process of recovery from breast cancer for younger and older patients: Changes during the first years. Cancer, 65, 1242-1254.

Vitaliano, P.P., Scanlan, J.M., Ochs, H.D., Syrjala, K., Siegler, I.C., \& Snyder, E.A. (1998). Psychosocial stress moderates the relationship of cancer history with natural killer cell activity. Annals of Behavioral Medicine, 20, 199-208.

Voogt, E., van der Heide, A., van Leeuwen, A.F., Visser, A.P., Cleiren, M.P., Passchier, J., et al. (2004). Positive and negative affect after diagnosis of advanced cancer. Psycho-Oncology, 14, 262-273.

Watson, D., Clark, L.A., \& Tellegen, A. (1988). Development and validation of brief measures of positive and negative affect: The PANAS scales. Journal of Personality and Social Psychology,

$545 \quad 54,1063-1070$

Wells, J.D., Hobfoll, S.E., \& Lavin, J. (1999). When it rains, it pours: The great impact of resource loss compared to gain on psychological distress. Personality and Social Psychology Bulletin, 25, $1172-1182$.

Zautra, A.J., Smith, B., Affleck, G., \& Tennen, H. (2001). Examination of chronic pain and affect relationship: Application of a dynamic model of affect. Journal of Consulting and Clinical Psychology, 69, 786-795.

Zautra, A.J., Johnson, L.M., \& Davis, M.C. (2005). Positive affect as a source of resilience for women in chronic pain. Journal of Consulting and Clinical Psychology, 73, 212-220. 